\title{
Tryptophan depletion and its implications
}

\section{for psychiatry}

\author{
CAROLINE BELL, JOLANE ABRAMS and DAVID NUTT
}

\begin{abstract}
Background Over the past 10 years the technique of tryptophan depletion has been used increasingly as a tool for studying brain serotonergic systems.
\end{abstract}

\begin{abstract}
Aims To review the technique of tryptophan depletion and its current status as a tool for investigating psychiatric disorders.
\end{abstract}

Method Systematic review of preclinical and clinical studies.

Results Tryptophan depletion produces a marked reduction in plasma tryptophan and consequently brain serotonin $(5-\mathrm{HT})$ synthesis and release. In healthy volunteers the effects of tryptophan depletion are influenced by the characteristics of the subjects and include some mood lowering, some memory impairment and an increase in aggression. In patients with depression tryptophan depletion tends to result in no worsening of depression in untreated subjects but a relapse in those who have responded to antidepressants (particularly serotonergic agents). In panic disorder the results are similar.

Conclusions The findings that tryptophan depletion produces a relapse of symptoms in patients with depression and panic disorder who have responded to treatment with antidepressants suggests that enhanced $5-\mathrm{HT}$ function is important in maintaining response in these conditions.

\section{Declaration of interest C.B., S.A.} and D.N. received honoraria and research grants from several pharmaceutical companies.
Serotonin (5-HT) is known to play an important role in a wide variety of functions, including mood, anxiety, aggression, sleep, appetite and sexual function, although the precise details of the mechanisms involved in these processes have not been established clearly. The situation has been hampered by the fact that until very recently it has not been possible to measure brain 5-HT directly, which means that we have had to rely on evidence from indirect sources. Over the past 10 years, neurotransmitter depletion paradigms have provided another means of examining the systems involved, and tryptophan (TRP) depletion in particular has emerged as an important tool for investigating 5-HT function. This review discusses the evolution of the technique of TRP depletion, the effects that it produces in healthy volunteers and patients suffering from different disorders, and what this tells us about the role of $5-\mathrm{HT}$ in the pathogenesis and treatment of several psychiatric conditions.

\section{THE TECHNIQUE OF TRYPTOPHAN DEPLETION}

The aim of TRP depletion is to lower brain 5-HT by depleting the body of its amino acid precursor TRP.

Figure 1 illustrates the processes in volved in the transport of TRP into the brain and its synthesis into 5-HT. A key factor in this is the level of TRP in plasma, which depends on the balance between the dietary intake of TRP and its removal from the plasma as a result of protein synthesis. Most of the TRP in plasma is proteinbound, with only about $5 \%$ being left free and available for transport into the central nervous system (CNS). It is this free TRP that is transported into the brain across the blood-brain barrier by an active protein shuttle for which five other large neutral amino acids (LNAAs: valine, leucine, isoleucine, phenylalanine and tyrosine) also compete. Once in the brain, TRP can take part in 5-HT synthesis, which is a two-step process. Tryptophan is first converted into 5 -hydroxytryptophan (5-HTP) by the enzyme tryptophan hydroxylase; 5-HTP is then decarboxylated by the enzyme aromatic acid decarboxylase to 5-hydroxytryptamine (5-HT). Tryptophan hydroxylase is only about $50 \%$ saturated in the CNS, which means that the rate of 5-HT synthesis is dependent on the availability of its substrate, free plasma TRP. This is therefore the rate-limiting step.

From this description and Fig. 1 it can be seen that three factors are important in determining the rate of 5-HT synthesis: the total amount of free plasma TRP; how much of that free TRP crosses the blood-brain barrier; and the activity of the tryptophan hydroxylase enzyme. Synthesis of 5-HT can be influenced by interfering with any or all of these factors. Early studies in the 1970s inhibited tryptophan hydroxylase using the drug parachlorophenylalanine and reported that this produced a relapse of depressive symptoms in patients who had previously responded to treatment with imipramine and tranylcypromine. Parachlorophenylalanine is, however, too toxic for ethical use in human subjects today, which has meant that interest has focused on techniques affecting the first two factors. As discussed above, free plasma TRP levels vary with the amount of dietary TRP and the rate of protein synthesis. Completely removing TRP from the diet reduces plasma TRP by only $15-20 \%$ and has few behavioural consequences. A much bigger fall in plasma TRP can be achieved by giving subjects an amino acid load that does not contain TRP, in the form of a drink. This has two effects: it stimulates protein synthesis in the liver, which uses up plasma TRP; and the amino acids that are given include the other LNAAs, which compete with TRP for transport across the blood-brain barrier and thus restrict the entry of TRP into the brain. Techniques that influence both of these factors have been shown to produce maximal brain TRP depletion: that is, using a combination of a low-protein (low-TRP) diet and a TRP-deficient protein load containing large amounts of the other LNAAs (reviewed in Reilly et al, 1997).

The TRP depletion paradigm is based on the hypothesis that reducing plasma TRP produces a consequent reduction in brain 5-HT synthesis and release. This has been confirmed in animals, with a large body of preclinical data showing that 


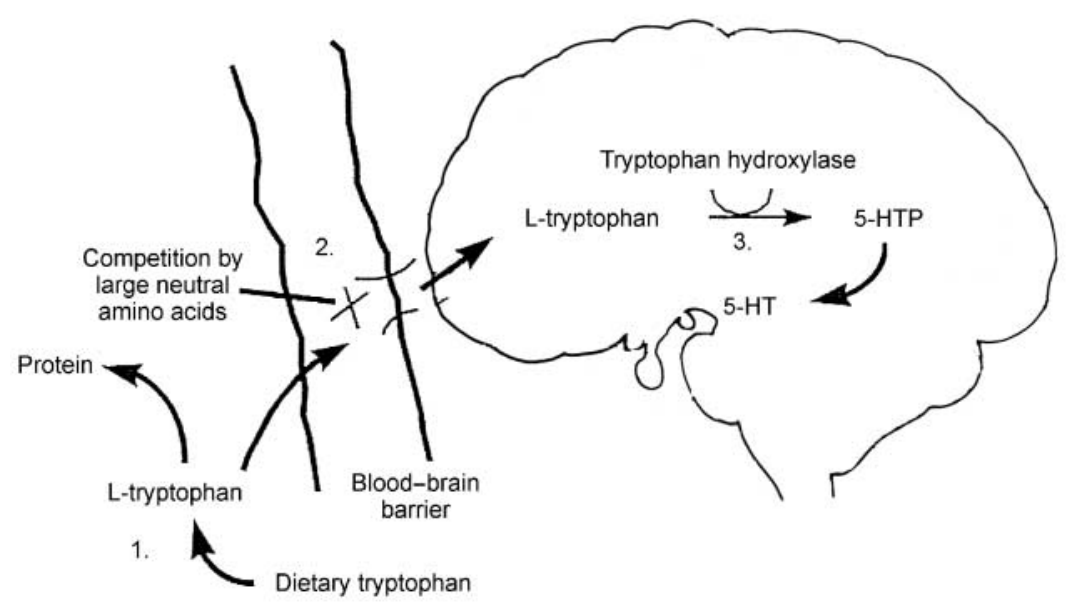

Fig. I Points at which serotonin (5-HT) synthesis may be controlled: (I) tryptophan availability: dietary restriction; (2) active transport: competition for the large neutral amino acid protein shuttle; (3) synthesis: inhibition of tryptophan hydroxylase. Best tryptophan depletion results are achieved using a combination of control at points I and 2. 5-HTP, 5-hydroxytryptophan.

depletion of TRP specifically reduces brain 5 -HT content and function. Ingestion of TRP-free amino acid mixtures in laboratory animals leads to extremely rapid changes in plasma TRP and brain 5-HT content, with maximal reductions of brain 5-HT occurring within $2 \mathrm{~h}$. It also alters behavioural indices of 5-HT function (increasing pain sensitivity, acoustic startle, motor activity and aggression, and reducing rapid eye movement (REM) sleep), effects that are reversed once brain TRP is restored. In monkeys, TRP depletion has similar effects, decreasing plasma TRP and cerebrospinal fluid (CSF) TRP and 5-hydroxyindoleacetic acid (5-HIAA).

In humans, the effects of TRP depletion on brain 5-HT have been more difficult to establish. Neuroendocrine studies have shown that TRP depletion produces postsynaptic receptor supersensitivity, presumably secondary to reduced synaptic availability of 5-HT. Recent studies in healthy volunteers involving continuous CSF sampling have shown that TRP depletion reduces central TRP and 5-HIAA levels by $80-90 \%$ and $24-40 \%$, respectively (Carpenter et al, 1998; Williams et al, 1999). The time course of these responses was also slightly delayed compared with the plasma findings, with the nadir for CSF TRP being 7-10 h after TRP depletion and even longer (12-14 h) for 5-HIAA. One study of the rate of 5-HT synthesis in vivo using positron emission tomography (PET) has reported that TRP depletion reduces the rate of synthesis by up to $40 \%$ of baseline values (Nishizawa et al, 1997).
Although the details vary, a common procedure for TRP depletion involves two test days about 1 week apart in a doubleblind, crossover design. On both occasions subjects follow a low-TRP (160 mg/day) diet for $24 \mathrm{~h}$ prior to the test day, followed by an overnight fast extending throughout the test day. On one test day, subjects consume a drink containing a 100-g load of 15 amino acids (including the LNAAs described above) that does not contain TRP, and on the other they have a nutritionally balanced drink that does contain TRP. With this technique, free (and total) plasma TRP levels are reduced by about $80 \% 5-7 \mathrm{~h}$ after the depleting drink (Delgado et al, 1990; Williams et al, 1999). Behavioural measures are recorded throughout the test day, with peak effects usually being seen 5-7 $\mathrm{h}$ after TRP depletion (i.e. at the point of maximum depletion of plasma TRP).

\section{CLINICAL EFFECTS OF TRYPTOPHAN DEPLETION}

\section{Healthy volunteers}

The known link between 5-HT and affect led to the early studies of TRP depletion in healthy volunteers, focusing particularly on mood effects. They used male subjects exclusively and showed that TRP depletion produced significant mood lowering (although never amounting to clinical depression) in these groups (Young et al, 1985; Smith et al, 1987). Subsequent studies, however, have tended to report that TRP depletion results in no mood effects
(Benkelfat et al, 1994; Carpenter et al, 1998; Knott et al, 1999). The discrepancy between these findings is probably explained by differences in the baseline mood states of subjects in the studies: those that reported a mood-lowering effect used volunteers with mean baseline depression scores at the upper end of the normal range, whereas the more recent negative findings were obtained with fully euthymic subjects. Studies that have included women have reported a gender difference, with a tendency for TRP depletion to produce mood lowering in healthy women but not men, despite similar degrees of plasma TRP depletion. This suggests that women may be more predisposed to the effects of TRP depletion than men. This is interesting given the increased incidence of depression in women and evidence from PET imaging studies suggesting that there may be gender differences in 5-HT metabolism with TRP depletion producing greater biochemical effects in women than in men (Nishizawa et al, 1997).

An increased vulnerability to mood alteration during TRP depletion has been reported in male and female euthymic subjects who have a family history of affective illness (Benkelfat et al, 1994; Klaassen $e t$ al, 1999), although this finding has not been replicated by all studies (Ellenbogen $e t$ al, 1999). This suggests that a subgroup of patients might have a trait that makes them particularly vulnerable to alterations in $5-\mathrm{HT}$ function. It also fits with clinical experience that a family history of affective disorder is a predisposing factor for depression.

Studies examining the effects of TRP depletion on anxiety symptoms in healthy volunteers have reported no significant effects on anxiety (Klaassen et al, 1998). It does, however, produce an exacerbation in anxiety (although never amounting to panic) when TRP depletion is combined with pharmacological panicogenic challenges such as yohimbine (Goddard et al, 1995) and $\mathrm{CO}_{2}$ (Klaassen et al, 1998), which are known to induce panic symptoms in patients with untreated panic disorder.

Other studies that have focused on the memory and cognitive effects of TRP depletion in human subjects have shown that TRP depletion specifically impairs long-term memory formation and interferes with the process of memory consolidation (Schmitt et al, 2000). Tryptophan depletion has also been reported to result in an improvement in measures of focused attention (Schmitt et al, 2000), which may be a result of the inhibitory effect of 5-HT on 
other neurotransmitters, particularly those involved with attentional processes (noradrenaline and acetylcholine). Interestingly, TRP depletion does not produce changes in other measures of frontal functioning (Schmitt et al, 2000).

The role of 5-HT in aggressive behaviour has been the subject of much research. Decreased central 5-HT function has been implicated in impulsive aggressive acts, including violent offending, arson and suicide. Animal studies have confirmed these findings and have shown that reducing 5-HT function increases measures of aggression. Studies in humans have shown that in healthy volunteers TRP depletion produces a marked rise in the ratings of aggression during provocation in subjects with hightrait aggression but has little effect in those with low-trait aggression (Bjork et al, 2000).

The role of 5-HT in sleep is complex. It increases the number of wakings during the night and results in changes to sleep architecture, i.e. the suppression of REM and slow-wave sleep. A large body of work has examined the sleep findings seen in depression. The classical abnormalities reported include measures of sleep continuity (difficulty getting off to sleep, waking during the night and waking early in the morning) and architecture (reduced REM latency and an increased duration of the first REM period). It was hypothesised that TRP depletion in healthy volunteers would produce a reduction in 5-HT function that would be reflected by changes to the sleep electroencephalogram (EEG), possibly producing changes similar to those seen in patients with depression. One study reported findings consistent with this, that is, reduced REM latency after TRP depletion (Bhatti et al, 1998), although another study reported no change to REM latency or amount (Voderholzer et al, 1998).

\section{Depression (see Table I)}

Most of the studies of TRP depletion have been in the field of depression. This reflects the intense interest over the past 30 years in the involvement of 5-HT in the pathogenesis and treatment of affective disorders. At present, the evidence from untreated patients suggests that 5-HT function is reduced in depression. The evidence from treated patients suggests that antidepressants work in depression by increasing 5-HT and/or noradrenaline neurotransmission. Despite this knowledge, the site of the abnormality/ abnormalities causing the 5-HT dysfunction in depression and the precise mechanisms of action of antidepressants have not been established. The hypotheses include factors such as the level of TRP availability, the rate of 5-HT synthesis, reuptake or metabolism, the functioning of pre- or post-synaptic 5 -HT receptors and an altered interaction between 5-HT and other neurotransmitter systems. Tryptophan depletion provides a way of testing some of these theories.

The effects of TRP depletion in untreated depression were somewhat unexpected. It was hypothesised that if decreased 5-HT function were associated with depression, then TRP depletion would lead to a transient worsening of symptoms. In a seminal study, 43 patients with untreated depression underwent TRP depletion (Delgado et al, 1994). This was found to result in no further mood lowering over the course of the day but, interestingly, a bimodal response the next day, with $37 \%$ describing an improvement in symptoms and $23 \%$ a worsening. The lack of effect of TRP depletion in causing a worsening of depressive symptoms in untreated patients has been replicated by two other studies (Price $e t$ al, 1997, 1998). One explanation for this is that 5-HT function is already so diminished that further lowering has little effect on symptom exacerbation. It may be, however, that deficient 5-HT function is not the primary or sole cause of depression and may be the result of dysfunction in regions or circuits in the brain that are modulated by 5-HT. This would mean that altering 5-HT function by itself would not be expected to produce immediate effects. The improvement in symptoms on the day after TRP depletion has been replicated by one study (Price $e t$ $a l, 1998)$. The explanation for this is that TRP depletion results in an up-regulation of post-synaptic 5-HT receptors because of the decreased release of 5-HT at the synapse. When 5-HT levels are restored (by the next day), the net effect is an enhancement of 5-HT function, resulting in the improvement of symptoms.

Table I Effects of tryptophan depletion in depression

\begin{tabular}{|c|c|c|c|}
\hline Mood state & Subjects (men, women) & Findings & Reference \\
\hline \multirow[t]{3}{*}{ Untreated depression } & $43(21,22)$ & No mood effects & Delgado et al (1994) \\
\hline & $22(6,16)$ & No mood effects & Price et al (1997) \\
\hline & $38(17,21)$ & No mood effects & Price et al (1998) \\
\hline \multirow[t]{6}{*}{ Remitted on antidepressants } & $\begin{array}{l}\text { 2I (8, I3) (on SSRIs, TCAs, } \\
\text { MAOIs, lithium) }\end{array}$ & $|4 / 2|$ relapsed & Delgado et al (1990) \\
\hline & $20(\mathrm{II}, 9)$ (on citalopram) & $5 / 12$ relapsed with TRP depletion & Åberg-Wistedt et al (1998) \\
\hline & & $0 / 8$ relapsed with no TRP depletion & \\
\hline & $2 I$ (II, I0) (on SSRIs) & $7 / 21$ relapsed & Bremner et al (1997) \\
\hline & $15(9,6)$ (on desipramine) & $8 / 15$ relapsed & Delgado et al (1999) \\
\hline & I5 $(9,6)$ (on fluvoxamine) & 1/15 relapsed & \\
\hline \multirow[t]{4}{*}{ Remitted on no medication } & 15 (all women) & 10/15 relapsed & Smith et al (1997) \\
\hline & $14(5,9)$ & No mood lowering & Leyton et al (1997) \\
\hline & $12(4,8)$ & Mood lowering & Moreno et al (1999) \\
\hline & 12 controls $(4,8)$ & No mood lowering & \\
\hline
\end{tabular}

SSRIs, selective serotonin reuptake inhibitors; TCAs, tricyclic antidepressants; MAOIs, monoamine oxidase inhibitors; TRP, tryptophan. 
The role of 5-HT in the mechanism of action of antidepressants in depression has been examined extensively using TRP depletion. The hypothesis for these studies was that if, as proposed, a time-dependent process of neuronal adaptation led to an enhancement of 5-HT neurotransmission after long-term antidepressant treatment, then acute depletion would be expected to rapidly reverse the beneficial effects. This has now been tested in multiple studies that have examined the effects of TRP depletion in patients who had responded to antidepressant treatments (particularly serotonergic antidepressants) (Table 1) (Delgado et al, 1990; Bremner et al, 1997; Åberg-Wistedt et al, 1998; Leyton et al, 2000). These showed that rapid TRP depletion led to a return of depressive symptoms in patients who had responded to treatment with antidepressants. Interestingly, the content of the depressive symptomatology was often very similar to the original presentation of the illness and typically occurred about 4-6 $\mathrm{h}$ after the depleting drink (i.e. at the time of peak depletion of plasma TRP).

The rates of relapse from these studies (as measured by an increase in depressive symptoms on the Hamilton Rating Scale for Depression, HRSD; Hamilton, 1960) were consistently higher for those on serotonergic drugs compared with those receiving more noradrenergic agents. One study that specifically examined this difference reported that $53 \%$ of patients who had responded to fluoxetine relapsed compared with $7 \%$ of patients who had responded to treatment with desipramine (Delgado et al, 1999). In contrast, catecholamine depletion studies (which use $\alpha$-methyl- $p$-tyrosine to block the enzyme tyrosine hydoxylase and thus the synthesis of noradrenaline and dopamine) result in a similar transient depressive relapse in patients who had been treated successfully with noradrenergic drugs but not in those on serotonergic antidepressants (Miller et al, 1996). This suggests that increasing 5-HT or noradrenaline neurotransmission is important for the specific function of different antidepressants.

The rates of relapse for patients on selective serotonin reuptake inhibitors (SSRIs) ranged in the different studies from $80 \%$ (Delgado et al, 1991) in patients remitted for at least 2 weeks, to only $30 \%$ (Bremner et al, 1997) in patients remitted for an average of 45 weeks. This suggests that the TRP depletion-induced relapse may be more likely in recently remitted patients compared with those who have been treated for longer periods of time. It may be that increasing synaptic 5-HT is only the first step in a cascade of events that lead to adaptive changes in 5-HT neurons, and that disrupting 5-HT function at this later stage has less effect.

The time course of the effects of depletion paradigms (i.e. over hours) implies that synaptic availability of 5-HT is more important than post-synaptic receptor effects, which would not be expected to change as rapidly. This is quite a major shift in thinking, because previous teaching has suggested that the delay in the clinical effect of antidepressants is explained by changes to postsynaptic receptors and other processes downstream of the synapse.

Two studies have used PET imaging to examine the effects of TRP depletion in remitted patients. Both reported that TRP depletion resulted in a decrease in brain metabolism in the areas of the brain implicated in the pathogenesis of depression (i.e. dorsolateral prefrontal and orbitofrontal cortex, amygdala and thalamus) (Bremner et al, 1997; Morris et al, 1999). Interestingly, in one study changes were seen only in 7/21 patients and were limited to those who relapsed as a result of TRP depletion (Bremner et al, 1997).

Patients who have had previous episodes of depression are known to be vulnerable to further episodes and it might be expected that TRP depletion in this group would result in relapse of depressive symptoms. Three studies have examined this specifically, using subjects with a past history of depression who at the time were well and on no medication (Leyton et al, 1997; Smith et al, 1997; Moreno et al, 1999). Two of the three studies reported greater rates of depressive relapse after TRP depletion than after the control drink (Smith $e t$ al, 1997; Moreno et al, 1999). These findings imply that deficient 5-HT function may play a part in triggering depressive episodes in patients with a vulnerability or susceptibility to changes in 5-HT function.

The sleep effects of TRP depletion in patients with remitted depression are interesting. One study has reported that although none of a group of ten men remitted for 2-13 months relapsed during TRP depletion, their sleep EEGs did regain some of the abnormalities characteristic of depression (i.e. reduced sleep and REM latencies and increased REM percentage and density) (Moore et al, 1998). This suggests that sleep may be a sensitive index of TRP levels.
Seasonal affective disorder (SAD) is a subtype of mood disorder characterised by recurrent major depressive episodes that occur regularly in the autumn and winter and respond to light therapy. The pathophysiology of SAD and the mechanism of action of light therapy are still poorly understood, but several lines of evidence suggest that 5-HT may be important (Joseph-Vanderpool et al, 1993). The results of TRP depletion in this group are similar to those reported with depression. Untreated patients are the least vulnerable to the mood-lowering effects of TRP depletion (Neumeister et al, 1997b). Patients with SAD who have been treated and are in remission after light therapy are likely to relapse after TRP depletion (Lam et al, 1996; Neumeister et al, 1997a). Long-term remitted patients produce mixed responses to TRP depletion, with one study reporting relapse rates of $73 \%$ (Neumeister et al, 1998) and another reporting no effect on symptoms (Lam et al, 2000).

\section{Anxiety disorders}

Serotonin neurotransmission is thought to play a central role in panic and anxiety in general. Perhaps the strongest evidence comes from treatment studies, which have shown that many of the disorders, including panic, social phobia and obsessivecompulsive disorder (OCD), are amenable to treatment with serotonergic drugs such as the SSRIs (discussed by Bell \& Nutt, 1998).

Most TRP depletion studies in anxiety have been performed in panic disorder and OCD, although there are ongoing studies in social phobia and generalised anxiety disorder.

\section{Panic disorder}

Patients with untreated panic disorder experience panic symptoms in response to panicogenic challenges (e.g. lactate, $\mathrm{CO}_{2}$, cholecystokinin-4, yohimbine or flumazenil), a reaction that is blocked by effective antidepressant treatment of the condition. Studies consistently show that in untreated patients TRP depletion alone produces no exacerbation of anxiety (Miller et al, 2000). However, when TRP depletion is combined with a panicogenic challenge it tends to result in an increase in anxiety and an increased rate of panic attacks (Miller et al, 2000), although one study reported a reduction in the expected number of panic attacks compared with normal 
controls (Schruers et al, 2000). We have reported recently on the effects of TRP depletion and a flumazenil challenge in patients who have responded to treatment with SSRIs (Nutt et al, 1999). We showed that $5 / 8$ patients panicked in response to flumazenil when they were TRP depleted, compared with $0 / 8$ on the control day. This suggests that enhanced synaptic 5-HT neurotransmission is important in maintaining the response to SSRIs in this condition; and when this is reduced (by TRP depletion), panic results (a finding similar to that reported in depression).

\section{Obsessive-compulsive disorder}

Serotonergic tricyclic antidepressants and SSRIs have been used successfully in the treatment of OCD. It was hypothesised that, as with depression and panic disorder, TRP depletion might be expected to exacerbate OCD in this group of patients. Four studies have examined the effects of TRP depletion in OCD in remitted patients either taking medication (Barr et al, 1994) or remitted and drug-free (Smeraldi et al, 1997; HuwigPoppe et al, 1999). These have all shown that TRP depletion produces no effect on OCD or Tourette symptoms, although some mood-lowering changes were reported. The difference in this response compared with the findings in depression and panic disorder suggests that the treatment of OCD may be less dependent on the synaptic availability of 5-HT than these other conditions, and more dependent on changes further downstream. Alternatively, it may be a reflection of the design of these studies, which did not combine TRP depletion with a challenge (as used in the panic disorder studies); and it may be that if this were done, TRP depletion would produce a relapse in symptoms.

\section{Eating disorders}

There is considerable evidence for a role for 5 -HT in the control of feeding behaviour, with a reduction in 5-HT function being shown to lead to impaired satiety and weight gain in animals. Dieting is known to lower 5-HT function in healthy women (Anderson et al, 1990) and to increase their prolactin responses to TRP (implying post-synaptic supersensitivity as a result of reduced synaptic availability). It has also been reported that women with a personal history of depression become more depressed during dieting than those without (Smith et al, 1999).

Bulimia nervosa has been linked with lowered 5-HT function. Studies in acutely ill untreated subjects with bulimia nervosa found increases in subjective ratings of the fear of fatness and binge eating as a result of TRP depletion (Kaye et al, 2000). A similar study in a group of ten patients in remission from the condition reported that TRP depletion produced an increase in the symptoms of fear of fatness, fear of losing control over eating and an increase in the urge to eat (Smith et al, 1999). In both untreated and treated patients with bulimia nervosa and those in remission, there was a vulnerability to the mood-lowering effects of TRP depletion.

To date, no TRP depletion studies have been performed in anorexia nervosa.

\section{Other disorders}

\section{Dementia}

Studies that examined the effects of TRP depletion on memory and related cognitive processes have provided some evidence that reducing 5-HT function through this technique impairs memory consolidation. This may be of particular relevance for disorders in which a deficit of 5-HT function is known to be linked with cognitive effects (e.g. Alzheimer's disease, where diminished 5-HT levels were found, at post-mortem). In a study that examined the effects of TRP depletion in patients with Alzheimer's disease, a significant impairment in cognitive functioning was reported, again suggesting that compromised serotonergic function (in combination with cholinergic deficit) may make an important contribution to the cognitive decline seen in this group of patients (Porter et al, 2000).

\section{Bipolar disorder}

A role for serotonin in mania has been hypothesised (Yatham et al, 1999), although to date no TRP depletion studies have been performed in patients with acute mania. In patients recovered from bipolar disorder, TRP depletion has been reported to have either no effect (Cassidy et al, 1998) or to cause a slight but insignificant (due to small sample size) relapse of mania (Cappiello $e t$ al, 1997).

\section{Schizophrenia}

There is some evidence that 5-HT may be at least partially responsible for the negative symptoms of schizophrenia. The effects of TRP depletion in schizophrenia, however, appear to be quite complex. In one study, TRP depletion exacerbated depressive symptoms and had no effects on positive or negative psychotic symptoms (D'Souza et al, 1994), whereas in another study it worsened negative symptoms (Sharma et al, 1997). In a recent study of neuroleptictreated patients with schizophrenia, TRP depletion resulted in an improvement on two measures of cognition (Speed and Comprehension of Language Processing and Wisconsin Card Sorting Test; Golightly et al, 1999).

\section{DISCUSSION}

Tryptophan depletion provides a way of examining the role of 5-HT in the pathophysiology of several psychiatric disorders and the mechanism of action of medication (antidepressants in particular). The responses produced by TRP depletion in different patient groups give us some information about the role of 5-HT in these disorders. Many questions remain unanswered but TRP depletion does provide an interesting and usable tool with which to examine the involvement of $5-\mathrm{HT}$ in these conditions.

\section{ACKNOWLEDGEMENT}

We thank Dr Spilios Argyropoulos for his help with revisions to this paper.

\section{REFERENCES}

Åberg-Wistedt, A., Hasselmark, L., Stain-

Malmgren, R., et al (1998) Serotonergic 'vulnerability' in affective disorder: a study of the tryptophan depletion test and relationships between peripheral and central serotonin indexes in citalopram-responders. Acta Psychiatrica Scandinavica, 97, 374-380.

Anderson, I. M., Parry-Billings, M. \& Newsholme, E. (1990) Dieting reduces plasma tryptophan and alters $5 \mathrm{HT}$ function in women. Psychological Medicine, 20, 785-791.

Barr, L. C., Goodman, W. K., McDouglie, et al (1994) Tryptophan depletion in patients with obsessivecompulsive disorder who respond to serotoninreuptake inhibitors. Archives of General Psychiatry, 5I, 309-317.

Bell, C. J. \& Nutt, D. J. (1998) Serotonin and panic. British Journal of Psychiatry, 172, 465-47I.

Benkelfat, C., Ellenbogen, M. A., Dean, P., et al (1994) Mood-lowering effect of tryptophan depletion: enhanced susceptibility in young men at genetic risk for major affective disorders. Archives of General Psychiatry, 5I, 687-697.

Bhatti, T., Gillin, J. C., Seifritz, E., et al (1998) Effects of a tryptophan-free amino acid drink challenge on normal human sleep electroencephalogram and mood. Biological Psychiatry, 43, 52-59.

Bjork, J. M., Dougherty, D. M., Moeller, F. G., et al (2000) Differential behavioral effects of plasma tryptophan depletion and loading in aggressive and 
nonaggressive men. Neuropsychopharmacology, 22, 357-369.

Bremner, J. D., Innis, R. B., Salomon, R. M., et al (1997) Positron emission tomography measurement of cerebral metabolic correlates of tryptophan depletion-induced depressive relapse. Archives of General Psychiatry, 54, 364-374.

Cappiello, A., Sernyak, M. J., Malison, R.T., et al (1997) Effects of acute tryptophan depletion in lithiumremitted manic patients: a pilot study. Biological Psychiatry, 42, 1076-1078

Carpenter, L. L., Anderson, G. M., Pelton, G. H., et al (1998) Tryptophan depletion during continuous CSF sampling in healthy human subjects. Neuropsychopharmacology, 19, 26-35.

\section{Cassidy, F., Murry, E. \& Carroll, B. J. (1998)}

Tryptophan depletion in recently manic patients treated with lithium. Biological Psychiatry, 43, 230-232.

\section{D'Souza, D., Gil, R. \& Abi-Draghjam, A. (1994)}

Tryptophan depletion paradigm: implications for schizophrenia. Biological Psychiatry, 35, 703.

Delgado, P. L., Charney, D. S., Price, L. H., et al (1990) Serotonin function and the mechanism of antidepressant action. Reversal of antidepressant induced remission by rapid depletion of plasma tryptophan. Archives of General Psychiatry, 47, 4II-4I8.

_ , Price, L. H., Miller, H. L., et al (199I) Rapid serotonin depletion as a provocative challenge test for patients with major depression: relevance to antidepressant action and the neurobiology of depression. Psychopharmacology Bulletin, 27, 321-330.

_, _ , , et al (1994) Serotonin and the neurobiology of depression: effects of tryptophan depletion in drug-free depressed patients. Archives of General Psychiatry, 5I, 865-874.

\section{_ , Miller, H. L., Salomon, R. M., et al (1999)}

Tryptophan-depletion challenge in depressed patients treated with desipramine or fluoxetine: implications for the role of serotonin in the mechanism of antidepressant action. Biological Psychiatry, 46, 212-220.

Ellenbogen, M. A., Young, S. N., Dean, P., et al (1999) Acute tryptophan depletion in healthy young women with a family history of major affective disorder. Psychological Medicine, 29, 35-46.

Goddard, A. W., Charney, D. S., Germine, M., et a (1995) Effects of tryptophan depletion on responses to yohimbine in healthy subjects. Biological Psychiatry, 38, 74-85.

Golightly, K., Lloyd, J. \& Hobson, J. (1999) Effect of tryptophan depletion on cognition in schizophrenia. journal of Psychopharmacology, 13, A54-OR2.

Hamilton, M. (1960) A rating scale for depression Journal of Neurology, Neurosurgery and Psychiatry, 23. $56-62$

Huwig-Poppe, C., Voderholzer, U., Backhaus, J., et a (1999) The tryptophan depletion test. Impact on sleep in healthy subjects and patients with obsessive-

compulsive disorder. Advances in Experimental Medicine and Biology, 467, 35-42.

Joseph-Vanderpool, J., Jacobsen, F. M., Murphy, D. L., et al (1993) Seasonal variation in behavioral responses to $\mathrm{m}$-CPP in patients with seasonal

affective disorder and controls. Biological Psychiatry, 33, $495-504$

Kaye, W. H., Gendall, K. A., Fernstrom, M. H. et al (2000) Effects of acute tryptophan depletion on mood in bulimia nervosa. Biological Psychiatry, 47. I5I-157.

\section{CLINICAL IMPLICATIONS}

Findings that tryptophan (TRP) depletion produces a relapse of symptoms in treated depression (and panic disorder) suggest that enhanced serotonin (5-HT) function is important in maintaining response to antidepressants.

- Findings that TRP depletion produces relapse or precipitates symptoms in patients with a personal or family history of affective disorders suggest that these subjects have a vulnerability to alterations in $5-\mathrm{HT}$ function.

- Lack of effect of TRP depletion in untreated depression and panic disorder and in healthy volunteers implies that $5-\mathrm{HT}$ dysfunction is not the primary or sole cause of these disorders.

\section{LIMITATIONS}

- We are not yet able to use TRP depletion as a tool to predict the likelihood of relapse when patients are taken off antidepressants.

- In humans it is difficult to establish the degree to which TRP depletion produces a reduction in brain $5-\mathrm{HT}$ and the time course of this response.

- There are only a small number of studies in conditions other than depression, which limits the conclusions that can be made about these conditions.

CAROLINE BELL, MRCPsych, JOLANE ABRAMS, BA, DAVID NUTT, FRCPsych, Psychopharmacology Unit, School of Medical Sciences, Bristol, UK

Correspondence: Caroline Bell, Psychopharmacology Unit, School of Medical Sciences, University Walk, Bristol BS8 ITD, UK

(First received 20 October 1999, final revision 31 October 2000, accepted 2 November 2000)

Klaassen, T., Klumperbeek, J., Deutz, N. E., et al (1998) Effects of tryptophan depletion on anxiety and on panic provoked by carbon dioxide challenge. Psychiatry Research, 77, 167-174.

_ , Riedel, W. J., van Someren, A., et al (1999) Mood effects of 24-hour tryptophan depletion in healthy firstdegree relatives of patients with affective disorders. Biological Psychiatry, 46, 489-497.

\section{Knott, V. J., Howson, A. L., Perugini, M., et al (1999)}

The effect of acute tryptophan depletion and fenfluramine on quantitative EEG and mood in healthy male subjects. Biological Psychiatry, 46, 229-238.

Lam, R.W., Zis, A. P., Grewal, A., et al (1996) Effects of rapid tryptophan depletion in patients with seasonal affective disorder in remission after light therapy. Archives of General Psychiatry, 53, 4I-44.

_ , Bowering, T. A., Tam, E. M., et al (2000) Effects of rapid tryptophan depletion in patients with seasonal affective disorder in natural summer remission. Psychological Medicine, 30, 79-87.

Leyton, M., Young, S. N., Blier, P., et al (1997) The effect of tryptophan depletion on mood in medicationfree, former patients with major affective disorder. Neuropsychopharmacology, 16, 294-297.

, Pihl, R. O., et al (2000) Effects on mood of acute phenylalanine/tyrosine depletion in healthy women. Neuropsychopharmacology, 22, 52-63.
Miller, H. E. J., Deakin, J. F. W. \& Anderson, I. M. (2000) Effect of acute tryptophan depletion on $\mathrm{CO}_{2}$ induced anxiety in patients with panic disorder and normal volunteers. British Journal of Psychiatry, I76, 182-188.

Miller, H. L., Delgado, P. L., Salomon, R. M., et a (1996) Clinical and biochemical effects of catecholamine depletion on antidepressant-induced remission of depression. Archives of General Psychiatry, 53, I17-128.

Moore, P., Gillin, C., Bhatti, T., et al (1998) Rapid tryptophan depletion, sleep electroencephalogram, and mood in men with remitted depression on serotonin reuptake inhibitors. Archives of General Psychiatry, 55, 534-539.

Moreno, F. A., Gelenberg, A. J., Heninger, G. R., et a (1999) Tryptophan depletion and depressive vulnerability. Biological Psychiatry, 46, 498-505.

Morris, J. S., Smith, K. A., Cowen, P. J., et al (1999) Covariation of activity in habenula and dorsal raphe nuclei following tryptophan depletion. Neuroimage, I0, 163-172.

Neumeister, A., Praschak, R. N., Hesselmann, B., et al (1997a) Effects of tryptophan depletion on drug-free patients with seasonal affective disorder during a stable response to bright light therapy. Archives of General Psychiatry, 54, 133-138. 
,et al (1997b) Rapid tryptophan depletion in

drug-free depressed patients with seasonal

affective disorder. American Journal of Psychiatry, 154

II53-II55.

_ , _ , _ , et al (1998) Effects of tryptophan depletion in fully remitted patients with seasonal affective disorder during summer. Psychological Medicine, 28, 257-264.

Nishizawa, S., Benkelfat, C., Young, S. N.,

et al (1997) Differences between males and females in rates of serotonin synthesis in human brain.

Proceedings of the National Academy of Sciences of the United States of America, 94, 5308-5313.

Nutt, D. J., Forshall, S., Bell, C., et al (1999)

Mechanisms of action of selective serotonin reuptake inhibitors in the treatment of psychiatric disorders. European Neuropsychopharmacology, 8 (suppl. 3),

S8I-S86.

Porter, R. J., Lunn, B. S., Walker, L. L., et al (2000) Cognitive deficit induced by acute tryptophan depletion in patients with Alzheimer's disease. American Journal of Psychiatry, 157, 638-640.

Price, L. H., Matison, R.T., McDougle, C. J., et al (1997) Neurobiology of tryptophan depletion in depression: effects of $\mathrm{m}$-chlorophenylpiperazine (mCPP). Neuropsychopharmacology, I7, 342-350.
_, _, _, et al (1998) The neurobiology of tryptophan depletion in depression: effects of intravenous tryptophan infusion. Biological Psychiatry, 43 339-347.

Reilly, J. G., McTavish, S. F. \& Young, A. H. (1997)

Rapid depletion of plasma tryptophan: a review of studies and experimental methodology. Journal of Psychopharmacology, II, 38I-392.

Schmitt, J., Jorissen, B. \& Sobczak, S. (2000)

Tryptophan impairs memory consolidation but improves focussed attention in healthy young volunteers. Journal of Psychopharmacology, 14, 21-29.

Schruers, K., Klaassen, T. \& Pols, H. (2000) Effects of tryptophan depletion on carbon dioxide-provoked panic in panic disorder patients. Psychiatry Research, 93 179-187.

Sharma, R. P., Shapiro, L. E., Kamath, S. K., et al (1997) Acute dietary tryptophan depletion: effects on schizophrenic positive and negative symptoms. Neuropsychobiology, 35, 5-10.

Smeraldi, E., Diaferia, G., Erzegovesi, S., et al (1997)

Tryptophan depletion in obsessive-compulsive patients. Biological Psychiatry, 40, 398-402.

Smith, K. A., Clifford, E. M., Hockney, R. A., et al

(1987) Effect of tryptophan depletion on mood in male and female volunteers: a pilot study. Human Psychopharmacology, I2, III-II7.

_ , Fairburn, C. G. \& Cowen, P. J. (1997) Relapse of depression after rapid depletion of tryptophan. Lancet, 349, 915-919.

_, _ \& _ (1999) Symptomatic relapse in bulimia nervosa following acute tryptophan depletion. Archives of General Psychiatry, 56, I7I-176.

Voderholzer, Y., Hornyak, M., Thiel, B., et al (1998) Impact of experimentally induced serotinin deficiency by tryptophan depletion on sleep EEG in healthy subjects. Neuropsychopharmacology, 18, 112-124.

Williams, W. A., Shoaf, S. E., Hommer, D., et al (1999) Effects of acute tryptophan depletion on plasma and cerebrospinal fluid tryptophan and 5-

hydroxyindoleacetic acid in normal volunteers. Journal of Neurochemistry, 72, 1641-1647.

Yatham, L. N., Shiah, I. S., Lam, R. W., et al (1999) Hypothermic, ACTH, and cortisol responses to ipsapirone in patients with mania and healthy controls. Journal of Affective Disorders, 54, 295-30I.

Young, S. N., Smith, S. E., Pihl, R. O., et al (1985) Tryptophan depletion causes a rapid lowering of mood in normal males. Psychopharmacology, 87, 173-177. 